
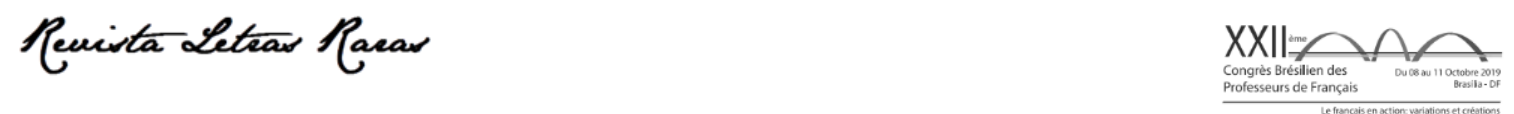

Actes du XXIlème Congrès brésilien des professeurs de français (dans Édition spéciale de la Revue Letras Raras 2020)

ISSN : 2317-2347 - v. 9, Dossier Spécial (2020)

Todo o conteúdo da RLR está licenciado sob Creative Commons Atribuição 4.0 Internacional

\title{
Les stages de FLE là où le français n'est pas présent dans
}

\section{l'enseignement officiel ${ }^{1}$}

\section{Ticiana Telles Melo*}

Doctorat en Sciences de l'Éducation à l'Université Fédérale du Ceará. Master en Linguistique à l'Université de l'État du Ceará. Licence en Lettres Portugaises et Françaises à l'Université Féderale du Ceará.

iD https://orcid.org/0000-0002-7438-0769

Reçu le 17 oct. 2020. Approuvé le 28 oct. 2020.

Comment citer cet article :

MELO, Ticiana Telles. Les stages de FLE là où le français n'est pas présent dans l'enseignement officiel. In: CONGRES BRESILIEN DES PROFESSEURS DE FRANÇAIS, 22., 2019, Brasília. Actes du XXIIème Congrès Brésilien des Professeurs de Français. Édition spéciale de la Revue Letras Raras: Campina Grande. EDUFCG. nov. 2020, p. 286-297.

\section{RÉSUMÉ}

Cet article a pour but de mettre en valeur l'importance des stages curriculaires pour la formation des futurs professeurs de Français Langue Étrangère et pour le dialogue avec la communauté touchée par les actions pédagogiques proposées par le stage. Nous nous sommes intéressées par la période comprise entre les années 2014 et 2017, motivés par la création et le développement du réseau Français sans Frontières, initiative de formation lingusitique et pédagogique qui regroupe plusieurs Institutions d'Enseigment Supérieur au Brésil. La situation étudiée nous a montré une réalité où le français ne fait pas partie des offres régulières de langue étrangère dans l'enseignement secondaire de la ville et les conséquences de cette réalité dans la construction de la maquette du cursus universitaire. De cette sorte, on a pu également observer les adaptations des disciplines de formation à l'appel actuel de l'internationalisation des études supérieures et leur impact sur les programmes de formation linguistique.

MOTS-CLÉS : Stage; Maquette curriculaire; Internationalisation des études supérieures, Français sous Objetctifs Universitaires.

\footnotetext{
${ }^{1}$ Communication présentée au XXIle Congrès Brésilien des Professeurs de Français de la Fédération Brésilienne des Professeurs de Français, en 2019, basée sur une analyse du sujet correspondant aux années 2014-2017 et publiée par l'auteure en langue portugaise sur la Revista Entrepalavras Volume 8.

$\triangle$ ticianatellesmelo@gmail.com
} 

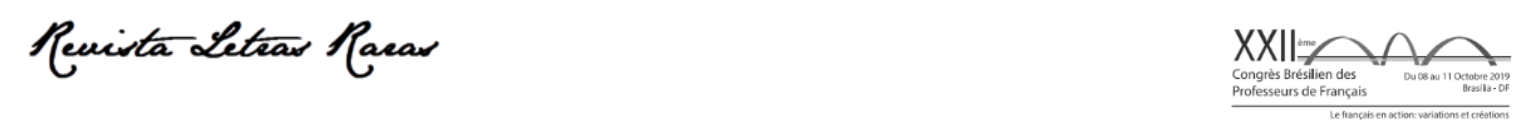

Actes du XXIlème Congrès brésilien des professeurs de français (dans Édition spéciale de la Revue Letras Raras 2020) ISSN : 2317-2347 - v. 9, Dossier Spécial (2020)

Todo o conteúdo da RLR está licenciado sob Creative Commons Atribuição 4.0 Internacional

\section{Introduction}

Avec ce travail, on vise à discuter les processus formatifs concernant les expériences de stage des étudiants du Cours de Lettres Portugaises et Françaises au sein de l'Université Fédérale du Ceará (UFC). Notre intérêt se concentre sur l'espace temporel instauré à partir du moment où cette institution a accueilli le Programme Francês sem Fronteiras du Ministère Brésilien de l'Éducation (MEC), en 2013. Avec ce programme, l'institution a commencé, à partir de 2014, à offrir des activités d'apprentissage du français destinées à ses étudiants (mais aussi à son cadre professionnel) en licence, master et doctorat, qui envisageaient une mobilité vers un pays francophone.

L'article sera divisé en deux parties: la première s'intéressera à la question de l'internationalisation des Institutions d'Enseignement Supérieur brésiliennes et la valorisation des études linguistiques pour la mobilité et la seconde présentera des questions pertinentes aux stages curriculaires et le versant de l'approche du Français sous Objectifs Universitaires (FOU) à partir de son double rapport avec l'internationalisation des études et la formation des futurs professeurs de Français Langue Étrangère.

\section{L'Internationalisation des Institutions d'Enseignement Supérieur au Brésil}

L'importance de l'internationalisation des Universités est l'un des fruits des transformations qu'ont subi ces institutions au fil des dernières années (SANTOS; ALMEIDA FILHO, 2012, p. 50). Ces transformations sont évidemment liées au mouvement sociétal dans lequel on vit, selon Morin (2000) depuis le XVI siècle on vivait dans l'ère planétaire, mais, c'est à la fin du XX siècle que l'on est entré dans la phase de la mondialisation.

Dans ce contexte de mondialisation des universités, formations diplômantes et études supérieures, il est incontournable que la participation à des réseaux internationaux, à des appels multilatéraux et la promotion d'une ambiance d'enseignement et de recherche sans frontières sont des critères, parmi d'autres, pour la définition du classement académique des universités. 

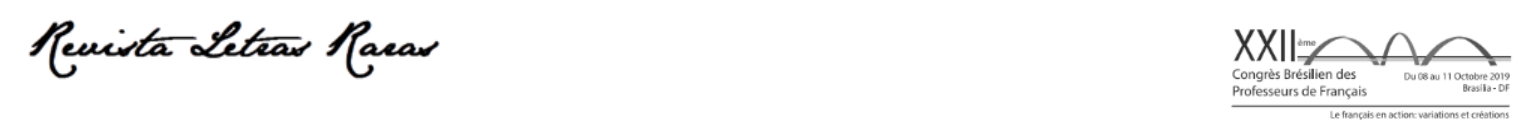

Actes du XXIlème Congrès brésilien des professeurs de français (dans Édition spéciale de la Revue Letras Raras 2020)

ISSN : 2317-2347 - v. 9, Dossier Spécial (2020)

Todo o conteúdo da RLR está licenciado sob Creative Commons Atribuição 4.0 Internacional

La mobilité (comme la mondialisation) est un concept qui se développe plutôt dans sa multiplicité, ce qui nous amène à préférer dire qu'il existe de différentes formes de mobilité. Pour la mobilité académique, on comprend qu'il y a une mobilité entrante et une mobilité sortante et que toutes les deux bâtissent le panorama de l'internationalisation de l'institution qui doit envisager que ses trois sphères - l'enseignement, la recherche et l'extensão - bénéficient de cette politique.

C'est exactement dans ce contexte polyvalent qui ont surgi les réflexions qui ont généré les études à propos du Français sous Objectifs Universitaires. Le FOU essaie de répondre aux spécificités langagières complexes qu'exige le milieu académique francophone. Aucun nouvel étudiant ne les maîtrise a priori, qu'il soit francophone ou allophone (COULON, 1997; GOES ET MANGIANTE, 2010, apud BOUCHET; VITAL; MACHADO, 2016).

Si on la compare à l'enseignement généraliste du français, cette approche se caractérise par des objectifs d'apprentissage plus précis, centrés sur la vie académique et la réussite du projet d'études. Elle se marque également par une période d'apprentissage beaucoup plus courte que l'habituelle et par une thématique très représentative de l'univers académique dans lequel les étudiants en situation de mobilité vont s'insérer.

Pour atteindre à ces objectifs, on exige de la part des enseignants une intimité avec des domaines de connaissances qui ne leur sont pas nécessairement familiers et, par manque de matériel didactique destiné à cette multitude de profils de mobilité et de candidats à la mobilité, la capacité à préparer leur propre matériel.

Un programme de préparation à la mobilité (FOU) suppose pour l'enseignantconcepteur des démarches institutionnelles et didactiques très différentes de ce que suppose un cours de Français Général (FG). La relation très étroite à des besoins externes conduit l'enseignant à collecter des données adaptées et à didactiser des supports spécifiques aux situations de mobilité (ALBUQUERQUECOSTA ; PARPETE, 2016, p. 49-50).

II faut souligner que FOU et FLE - ici compris comme le Français Généraliste (FG) - sont synchrones et ne sont pas excludents. Ce sont des approches qui visent à former les étudiants selon leur projet d'apprentissage. Et c'est pour cela que nous pensons que les cours de Lettres doivent élargir l'horizon des expériences formatives de leurs étudiants stagiaires et leur proposer des 

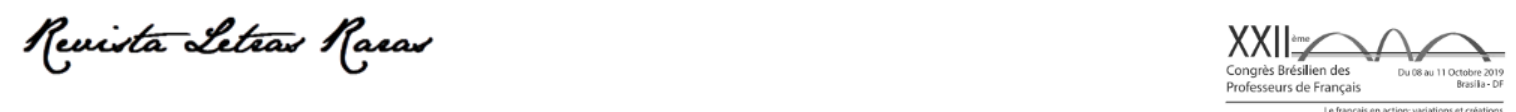

Actes du XXIlème Congrès brésilien des professeurs de français (dans Édition spéciale de la Revue Letras Raras 2020)

ISSN : 2317-2347 - v. 9, Dossier Spécial (2020)

Todo o conteúdo da RLR está licenciado sob Creative Commons Atribuição 4.0 Internacional

activités curriculaires qui puissent répondre tant au public non universitaire qu'aux exigences actuelles de la mobilité.

En plus, en ce qui concerne les stratégies et finalités didactiques, FLE et FOU partagent un même noyau dur, celui de l'approche actionnelle, pour la formation et la pratique des étudiants stagiaires c'est un atout considérable. Des auteurs comme Cuq et Gruca (2005) et Puren (2018a), parmi d'autres, affirment que la didactique du français est centrée sur l'approche actionnelle. Le Cadre Européen Commun de Références pour les Langues met l'emphase sur l'importance de l'exécution des tâches, surtout aux chapitres 2, 4 et 7 et considère les apprenants comme des acteurs sociaux qui:

[...] développent un ensemble de compétences générales et, notamment une compétence à communiquer langagièrement. Ils mettent en œuvre les compétences dont ils disposent dans des contextes et des conditions variés et en se pliant à différentes contraintes afin de réaliser des activités langagières permettant de traiter (en réception et en production) des textes portant sur des thèmes à l'intérieur de domaines particuliers, en mobilisant les stratégies qui paraissent le mieux convenir à l'accomplissement des tâches à effectuer. Le contrôle de ces activités par les interlocuteurs conduit au renforcement ou à la modification des compétences (CONSEIL DE L'EUROPE, 2003, p. 15).

On sait que le but final de l'apprentissage sous l'égide de l'approche actionnelle ne vise pas seulement la communication, mais le développement des activités interactives qui utilisent la langue étrangère. Dans le cas du FOU, les tâches finales doivent porter sur le monde de la mobilité. De l'apprenant, il peut être sollicité, par exemple, la compréhension écrite de la maquette du cours envisagé, la production d'une lettre de motivation, la compréhension orale d'une explication théorique de son domaine d'études ou sa capacité à s'exprimer sur le même sujet. La description d'un projet pédagogique faite par Puren, nous permet de dire que les enjeux de celui-ci s'accommodent facilement avec l'approche voulue par le FOU:

Un projet pédagogique, au contraire, a pour finalités premières l'autonomisation et la responsabilisation des apprenants, par ailleurs indispensables pour qu'ils se mobilisent sur un projet qui soit le leur : ce sont eux, par conséquent, qui doivent concevoir au départ l'action à réaliser à la fin de l'unité didactique, eux qui doivent rechercher, sélectionner et exploiter la documentation nécessaire, de sorte qu'il est impossible de prévoir à l'avance leurs besoins langagiers et culturels, et donc de préprogrammer l'exercisation (PUREN, 2018b, p. 2). 


\section{Revista Letras Racar}

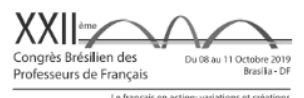

Actes du XXIlème Congrès brésilien des professeurs de français (dans Édition spéciale de la Revue Letras Raras 2020)

ISSN : 2317-2347 - v. 9, Dossier Spécial (2020)

Todo o conteúdo da RLR está licenciado sob Creative Commons Atribuição 4.0 Internacional

L'internationalisation des études supérieures et des institutions au Brésil a connu dans les années 2010 un essor unique. Le programme Francês sem Fronteiras intègre l'une des initiatives de mobilité internationale les plus importantes ayant lieu dans l'histoire de notre pays: le Programme Ciência sem Fronteiras - CsF (ABREU E LIMA, 2016). Pour en avoir une idée, ce programme, mis en place en 2011 par les ministères brésiliens de l'Éducation et de la Recherche, a financé 100000 bourses de mobilité entre 2011 et 2014 pour les étudiants de disciplines scientifiques (dont 10000 vers la France, le Québec et la Belgique). ${ }^{2}$

Dès les premiers appels du CsF, la fragilité de la compétence en langues étrangères de l'étudiant universitaire brésilien s'est démontrée. Visant à dépasser cette impasse, le MEC a créé le programme Inglês sem Fronteiras, qui s'est élargi vers d'autres langues, et a incorporé le nom de Idiomas sem Fronteiras. L'objectif de ce programme est la promotion "d'une politique linguistique pour l'internationalisation de l'Enseignement supérieur brésilien, tout en mettant en valeur la formation spécialisée des professeurs des langues étrangères"3.

Cette valorisation de la formation spécialisée des professeurs de langues comprend une nouvelle réalité formative qui demande à être incorporée si on veut préparer des professeurs pour le monde de la mobilité. Historiquement, les Cours de Lettres (si l'on pense aux formations en langues étrangères) ont déjà fait ce type de mouvement, lorsque de l'avènement du Français sous Objectif Spécifique - FOS (certaines formations l'appellent français instrumental, ou français à des fins de lecture académique) et de l'incorporation des technologies de l'information et de la communication pour l'éducation (TICEs). Le stage académique l'a déjà démontré que quand il intègre de nouveaux versants et produit de la recherche conséquente, on assiste à la promotion de la multiplication des possibilités professionnelles pour ses étudiants stagiaires.

\section{La formation professorale dans le Cours de Lettres à l'UFC}

Dans cette partie du texte on veut présenter une lecture de ce que c'est un curriculum, et discuter les disciplines de stages curriculaires dans la maquette du Cours de Lettres de l'UFC, pour

$2 \mathrm{http}: / /$ www.cienciasemfronteiras.gov.br/web/csf/o-programa. Consultation 25 mars 2018.

3 http://isf.mec.gov.br/index.php?option=com_content\&view=article\&id=69\&ltemid=674. Consultation 25 mars 2018. 


\section{Reuista Letraw Racar}

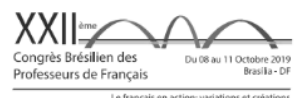

Actes du XXIlème Congrès brésilien des professeurs de français (dans Édition spéciale de la Revue Letras Raras 2020)

ISSN : 2317-2347 - v. 9, Dossier Spécial (2020)

Todo o conteúdo da RLR está licenciado sob Creative Commons Atribuição 4.0 Internacional

vérifier comment le FOU peut contribuer à la formation des futurs professeurs et proposer un autre parcours pour le stage qui doit confronter la situation assez particulière qui est celle de l'enseignement des langues dans les lycées à Fortaleza.

Pour comprendre le stage, on doit s'interroger sur ce que c'est un curriculum. Pour Silva (2002), le curriculum est une carte d'identité, sur laquelle s'inscrivent les parcours (délibérés ou non), les choix et les vécus qui font du sujet apprenant qui il est. Le curriculum formel est le résultat d'un choix auquel des contenus jugés appropriés ont été ajoutés et se font respecter et valoriser.

En ce qui concerne les parcours à se faire présents dans le curriculum de l'actualité, la mobilité peut représenter un atout majeur. De cette façon, la construction du curriculum ne doit pas exclure les savoirs venus du phénomène de l'internationalisation des études et de ses conséquences dans l'apprentissage des langues étrangères pour la constitution d'un champ méthodologique de la formation envisagée par les Cours de Lettres.

Le stage est le moment clé de la formation des étudiants des cours de formation pour futurs professeurs. Almeida et Pimenta (2015, p. 30, notre traduction) $)^{4}$, considèrent le stage comme un "espace/temps qui favorise la compréhension de ce qui est la mise en profession pour l'enseignement". Gautherot complète cette analyse en créant une espèce de symbiose entre le stage et le temps du vécu

Temps de la pause et de la permanence dans l'écoulement inéluctable du temps chronologique. [...] Temps de la connaissance, temps de la critique, temps de l'observation, temps du retour sur soi, temps de la déconstruction et de la reconstruction, temps de l'invention (GAUTHEROT, 1992, p. 52).

Les stages, moments partagés entre le professeur référent, les camarades, le personnel des écoles accueillantes, les lycéens, parmi d'autres acteurs, sont aussi interprétés socialmente comme un rituel de passage important pour la jeunesse. Ce rite (BOURDIEU, 1982) permettra la légitimation de l'entrée dans un champ privilégié qui est celui des diplômés, de ceux qui méritent la position conquise après des années d'études et d'autres investissements.

\footnotetext{
${ }^{4}$ Dans le texte original "O estágio coloca-se, então, como um espaço/tempo favorecedor da compreensão a respeito da profissionalidade docente."
} 


\section{Revista Letras Racar}

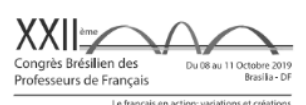

Actes du XXIlème Congrès brésilien des professeurs de français (dans Édition spéciale de la Revue Letras Raras 2020)

ISSN : 2317-2347 - v. 9, Dossier Spécial (2020)

Todo o conteúdo da RLR está licenciado sob Creative Commons Atribuição 4.0 Internacional

La maquette du Cours de Lettres Portugaises et Françaises de l'UFC présente deux disciplines obligatoires de stage en langue étrangère qui se font à la dernière année de formation. $\mathrm{Si}$ I'on considère le total des disciplines offertes par le Département de Lettres Étrangères, on comptabilise un autre composant préparatoire au métier qui est la discipline "Élaboration de matériel didactique en Français Langue Étrangère".

L'appropriation du Français sous Objectifs Universitaires a lieu dans les trois disciplines mentionnées. Cette réalité nous demande une nouvelle pratique de validation des expériences antérieures au stage curriculaire, ce qui va aérer le parcours académique de nos étudiants, une fois que notre matrice curriculaire présente encore une vision segmentée de la formation, destinant les derniers semestres aux pratiques professionnelles. Cette possibilité peut minorer la séparation conçue entre contenus disciplinaires et formation pédagogique et valoriser la pratique en tant que processus et non comme un résultat de la formation antérieure.

On sait qu'une structure curriculaire rigide n'apporte pas de bons fruits à la formation, surtout si elle organise la maquette d'études d'une façon à distancer les contenus dit disciplinaires des pratiques professorales. Soucieux de leur parcours, les étudiants cherchent de plus en plus à anticiper leur pratique professionnelle. L'équipe des professeurs travaille des formes de propositions d'un stage non curriculaire. On peut citer comme expériences réussies: la sélection des professeurs stagiaires pour des cours de Français destinés à la communauté non universitaire. Cette expérience, si suivi par un professeur référent et générant un rapport d'expérience, dialogue d'une façon intime avec le stage final du cours de Lettres.

L'intégration d'une formation professorale visant le FOU vient répondre en partie à cette promotion d'anticipation et diversification des stages curriculaires.

À l'Université Fédérale du Ceará, le premier stage se présente comme une discipline hybride de 64 heures et s'appelle Fondements Théoriques pour l'Enseignement de la Langue Française. Une partie du volume horaire est destinée à l'acquisition des concepts de base indispensables à l'expérience exigée au semestre suivant ; une autre partie est consacrée à l'observation que l'étudiant doit faire dans des cours de français de la ville. Comme tout stage, le semestre sera validé sous la composition d'un rapport de stage. 


\section{Revista Letras Racar}

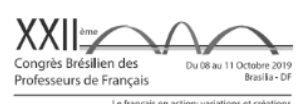

Actes du XXIlème Congrès brésilien des professeurs de français (dans Édition spéciale de la Revue Letras Raras 2020)

ISSN : 2317-2347 - v. 9, Dossier Spécial (2020)

Todo o conteúdo da RLR está licenciado sob Creative Commons Atribuição 4.0 Internacional

Les textes théoriques apportent la discussion à propos de l'enseignement des langues étrangères sous de divers aspects. La question de la politique linguistique et son rapport avec les différents moments sociohistoriques des communautés concernées est aussi présente. On discute divers sujets relatifs au champ des langues étrangères et le rôle de la langue française dans ce panorama. La présentation des principales méthodologies d'enseignement de langue pour arriver à l'approche actionnelle et ses caractéristiques nous permet de faire le pont avec le FOU. Une fois que FLE e FOU ont des principes similaires et se basent sur la construction d'un acteur social qui doit accomplir des actions - des interactions et de co-actions - grâce à la langue, la sensibilisation au FOU intéresse nombreux étudiants stagiaires.

À ce moment, grâce à l'observation, les stagiaires vont connaître la réalité matérielle dans laquelle ils vont s'insérer en tant que professeurs. La réflexion, l'échange fait avec le professeur qui les accueille au sein de son groupe sont indispensables à leur formation.

Enfin, les lectures, les discussions en salle et l'évaluation des rapports de stages nous permettent d'annoncer des résultats positifs. Les étudiants stagiaires se montrent de plus en plus motivés à travailler le FOU. La sollicitation à la construction de matériels didactiques innovants est, à notre avis, propre aux activités demandées par une discipline de cette caractéristique.

La discipline Élaboration de Matériels didactiques en Français Langue Étrangère, de 32 heures, vise à préparer l'étudiant à évaluer, élaborer et utiliser des matériels destinés à l'apprentissage du FLE. L'étudiant doit, donc, produire des matériels cohérents avec la proposition méthodologique actuelle. Les outils qui permettront le bon développement de cette discipline sont pertinents pour le FLE et pour le FOU. L'appropriation de ces éléments, l'activation des expériences préalables en tant qu'usagers de la langue française, mais aussi en tant que sujet professeur en formation, est l'objectif final de cette composante curriculaire. On voit donc que l'intégration du FOU dans cette discipline se fait d'une façon tranquille et favorise l'élaboration des dossiers qui peuvent composer le menu d'activités à être proposées lors d'un cours de FOU.

Avant de parler des expériences proposées par la discipline de stage pratique, il faut dire que la réalité de l'enseignement dans le réseau des lycées à Fortaleza est extrêmement précaire. À l'exception d'un seul lycée de l'enseignement public (et prévu pour les élèves de la première année), 

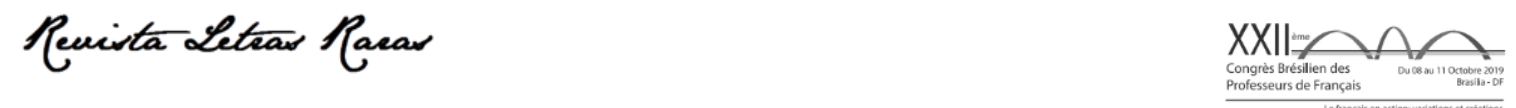

Actes du XXIlème Congrès brésilien des professeurs de français (dans Édition spéciale de la Revue Letras Raras 2020)

ISSN : 2317-2347 - v. 9, Dossier Spécial (2020)

Todo o conteúdo da RLR está licenciado sob Creative Commons Atribuição 4.0 Internacional

le français n'est pas une discipline qui fait pas partie de l'offre régulière des langues étrangères de l'enseignement secondaire.

Cependant, dans une politique de valorisation linguistique dans la formation des lycéens des écoles publiques, et avec le but "d'approfondir leurs connaissances des relations linguistiques et culturelles des langues étrangères modernes offertes dans le réseau éducatif"5 depuis 2017, le gouvernement de l'État a créé des centres de langues pour ce public de la capitale et de trois autres villes. Depuis 2019, le français est l'une des trois langues enseignées dans trois de ces centres de la capitale.

Ces données ont des rapports immédiats avec les offres de stage curriculaires proposées par l'Université Fédérale du Ceará. Les étudiants pour leur grande majorité sont amenés à faire leur stage dans des écoles (surtout professionnelles ou à plein temps) où le français est une des disciplines dites eletivas. Le caractère fragile de ce type de discipline ne permet pas une insertion de l'étudiant stagiaire dans l'école comme il serait convenable dans tout stage. Le stage pratiqué dans ces écoles ou dans les centres de langues se fait par le biais des cours supplémentaires d'un volume horaire variable. 6

Quand il y a l'impossibilité de faire le stage entre les murs d'une école, il y a cette même proposition de cours rapides de FLE assurés par des étudiants stagiaires à la communauté, via le minicours Descobrindo a Língua Francesa, qui dure un semestre et est basé sur l'approche actionnelle.

Un autre modèle de stage curriculaire existe traditionnellement au sein des Casas de Cultura Estrangeira de l'UFC. La Casa de Cultura Francesa a toujours accueilli nos étudiants et depuis trois ans s'occupe également de l'accompagnement formatif du stagiaire. II faut dire que ce projet d'extensão universitaire a déjà plus de 50 ans et a été créé avec le profil d'une école préparatoire pour les futurs professeurs, mais de nos jours il a la vocation de préparer la communauté universitaire et non universitaire aux compétences linguistiques en langues étrangères, avec une équipe des professeurs universitaires qui composent le plus grand projet d'extensão de notre

\footnotetext{
${ }^{5}$ www.seduc.ce.gov.br/centro-cearense-de-idiomas-cci. Consultation 30 septembre 2020.

6 Une première expérience de stage pour les étudiants stagiaires de français de l'UFC dans les centres de langue a eu lieu pendant le premier semestre 2020, bénéficiant 20 lycéens des trois centres où le français fait partie de l'offre linguistique.
} 

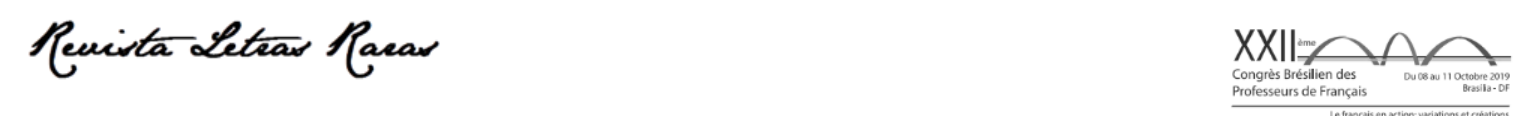

Actes du XXllème Congrès brésilien des professeurs de français (dans Édition spéciale de la Revue Letras Raras 2020) ISSN : 2317-2347 - v. 9, Dossier Spécial (2020)

Todo o conteúdo da RLR está licenciado sob Creative Commons Atribuição 4.0 Internacional

université. De cette collaboration, les stagiaires ont toujours la possibilité de faire leur stage dans ce centre d'excellence (GIRAUD, 2018).

Avec le mouvement d'internationalisation de l'UFC, dans le curriculum de l'actualité, la préparation à la mobilité est un atout majeur. De cette façon, le parcours conventionnel ne doit pas exclure les savoirs apportés par ce phénomène et ses conséquences dans l'apprentissage des langues étrangères pour la constitution d'un champ épistémologique de la formation valorisée par les cours de Lettres.

De cette façon, le FOU dans le cadre de l'IsF est l'une des possibilités pour les étudiants stagiaires. Pour y accéder, il faut se soumettre à la sélection et avoir les conditions minimales exigées, telle qu'une certification qui corresponde au niveau B1 du Cadre Européen Commun de Références pour les langues. Dans le contrat du boursier, il y a un volume horaire destiné aux études théoriques et pratiques de la méthodologie FOU. La méthodologie de travail est discutée par un groupe d'études qui représente un moment d'interaction entre les boursiers et d'autres étudiants, même ceux qui ne sont pas encore inscrits dans les disciplines de stage.

L'équipe des professeurs et stagiaires (curriculaires et volontaires) organise des cours FOU pour la communauté universitaire et la Coordination du Cours de Lettres peut valider cette expérience comme l'équivalent de la discipline de stage final. Le rapport des expériences qui nous sont parvenus montrent qu'une partie de l'enthousiasme de la réussite du stagiaire repose sur le fait du dialogue établi entre cette expérience et les deux disciplines (stage et élaboration de matériel) qui l'ont précédée.

\section{En guise de conclusion}

Le stage curriculaire de langue française à l'UFC doit faire face à beaucoup de défis et cherche à montrer sa vitalité grâce à des interlocutions faites avec plusieurs secteurs de la ville. Comme partenaires principaux, on cite trois sphères publiques de notre ville : l'école de l'enseignement secondaire, les Centres de Langues de l'État et la Casa de Cultura Francesa. On n'oublie pas que la demande particulière de l'UFC avec le FOU nous a permis d'élargir les 


\section{Revista Letras Racar}

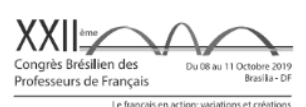

Actes du XXIlème Congrès brésilien des professeurs de français (dans Édition spéciale de la Revue Letras Raras 2020)

ISSN : 2317-2347 - v. 9, Dossier Spécial (2020)

Todo o conteúdo da RLR está licenciado sob Creative Commons Atribuição 4.0 Internacional

possibilités de stage, avec l'intégration de l'appel à la mobilité dans la formation du futur professeur de français.

La multiplicité de cette préparation doit avant tout permettre à l'étudiant stagiaire de reconnaître qu'un professeur de langues peut répondre à plusieurs appels formatifs de la société. De la part du professeur référent, on espère l'ouverture et la disponibilité pour la recherche de nouveaux locus à être occupés par les langues étrangères.

\section{Références}

ABREU E LIMA, D. M. O programa Inglês sem Fronteiras e a política de incentivo à internacionalização do Ensino Superior brasileiro. In: SARMENTO, S.; ABREU E LIMA, D. M.; MORAES FILHO, W. B. (Org.). Do Inglês sem Fronteiras ao Idiomas sem Fronteiras: a construção de uma política linguística para a internacionalização. Belo Horizonte: Editora UFMG, 2016.

ALBUQUERQUE-COSTA, H.; PARPETTE, C. (Orgs.). Français sur Objectif Universitaire: méthodologie, formation des enseignants et conception de programmes. São Paulo: Paulistana, 2016.

ALMEIDA, M. I.; PIMENTA, S. G. Centralidade do estágio em curso de Didática nas licenciaturas: rupturas e ressignificações. In: ALMEIDA, M. I.; PIMENTA, S. G. Estágios supervisionados na formação docente: educação básica e educação de jovens e adultos. São Paulo: Cortez, 2015.

BOUCHET, K.; VITAL, J.; MACHADO, R. Impact du type de mobilité étudiante sur les programmations en Français sur Objectif Universitaire. Le Français sur Objectif Universitaire: méthodologie, formation des enseignants et conception de programmes. Humanitas, v. 4, p.109-133, 2017. Disponible en: [https://docplayer.fr/159988701-Impact-du-type-de-mobilite-etudiante-sur-lesprogrammations-en-francais-sur-objectif-universitaire.html]. Consultation le: 23 sept. 2020.

BOURDIEU, P. Ce que Parler Veut Dire: L'Économie des Échanges linguistiques. Paris: Fayard, 1982.

CONSEIL DE L'EUROPE: Cadre Européen Commun de Référence pour les langues: Apprendre, enseigner, évaluer, 2003. Disponible en:[https://rm.coe.int/16802fc3a8]. Consultation le: 23 sept. 2020.

CUQ, J-P.; GRUCA, I. Cours de didactique du français langue étrangère et seconde. Grenoble: PUG, 2005. 

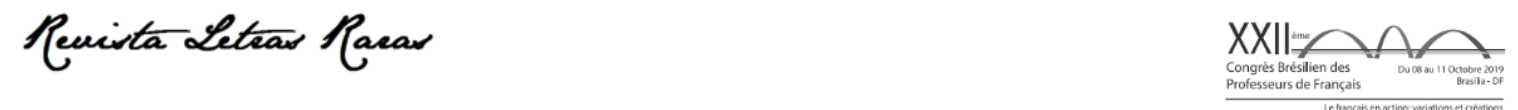

Actes du XXIlème Congrès brésilien des professeurs de français (dans Édition spéciale de la Revue Letras Raras 2020)

ISSN : 2317-2347 - v. 9, Dossier Spécial (2020)

Todo o conteúdo da RLR está licenciado sob Creative Commons Atribuição 4.0 Internacional

GIRAUD, A.C.B. (Org.) Casa de Cultura Francesa 50 Anos: Memória e Saber. Fortaleza: Edições UFC, 2018.

GAUTHEROT, J.-M. Du bon et du mauvais usage des stages. Le Français dans le Monde: recherches et applications, Paris, n.12, p. 52-59, 1992.

MORIN, E. Os Sete Saberes Necessários à Educação do Futuro. São Paulo: Cortez, 2000.

PUREN, C. L'Actualité de l'Approche Communicative dans le Cadre de la Mise en Oeuvre de la Perspective Actionnelle : Une Affaire de Construction Situtée et Finalisée, 2018a. Disponible en: [https://www.christianpuren.com/mes-travaux/2018f/]. Consultation le: 30 sept. 2020.

PUREN, C. Comment Intégrer la Démarche de Projet dans le Travail en Classe sur les Unités Didactiques des Manuels de Langue, 2018b. Disponible en: [https://www.christianpuren.com/app/download/13569365127/069_Unit\%C3\%A9s_didactiques_manu els_compatibilit\%C3\%A9_d\%C3\%A9marche_projet.pdf?t=1529139260]. Consultation le: 7 oct. 2020.

SANTOS, F. S.; ALMEIDA FILHO, N. A quarta missão da universidade: internacionalização universitária na sociedade do conhecimento. Brasília: Editora Universidade de Brasília; Coimbra: Imprensa da Universidade de Coimbra, 2012.

SILVA, T. T. Documentos de identidade: uma introdução às teorias do currículo. Belo Horizonte: Autêntica, 2002. 